\title{
Underwater Camera Calibration Using Wavelength Triangulation
}

\author{
Timothy Yau \\ University of Alberta \\ Edmonton, AB, Canada \\ thyau@ualberta.ca
}

\author{
Minglun Gong \\ Memorial University of Newfoundland \\ St. John's, NL, Canada \\ gong@cs.mun.ca
}

\author{
Yee-Hong Yang \\ University of Alberta \\ Edmonton, AB, Canada \\ yang@cs. ualberta.ca
}

\begin{abstract}
In underwater imagery, the image formation process includes refractions that occur when light passes from water into the camera housing, typically through a flat glass port. We extend the existing work on physical refraction models by considering the dispersion of light, and derive new constraints on the model parameters for use in calibration. This leads to a novel calibration method that achieves improved accuracy compared to existing work. We describe how to construct a novel calibration device for our method and evaluate the accuracy of the method through synthetic and real experiments.
\end{abstract}

\section{Introduction}

Underwater stereo camera systems have been used for many years in applications such as marine biology and archaeology, and are becoming more common as the technology improves. One of the main difficulties for computer vision is refraction caused by the camera housing. This introduces distortions in the image that depend on the scene distance and cannot be modelled exactly as a radial distortion, but are often approximated as such [10,11].

Recently there has been increasing interest in applying a physically correct model of refraction to improve the accuracy of stereo reconstructions $[1,5,6,11]$. Agrawal et al. provided important new insights into the properties of this model that greatly simplify the calibration procedure compared to earlier efforts [1]. Building upon these results, we further characterize the flat refraction camera model by studying the dispersion of light, which is the phenomenon where light refracts at a different angle depending on its wavelength. While previous authors regarded dispersion as a minor problem to be ignored [9], we show that the disparate light paths can be exploited in a manner similar to triangulation, thereby increasing calibration accuracy.

In this paper we first show that dispersion provides additional constraints on the parameters of the refraction model, and explain how they can be used in calibration. Next we

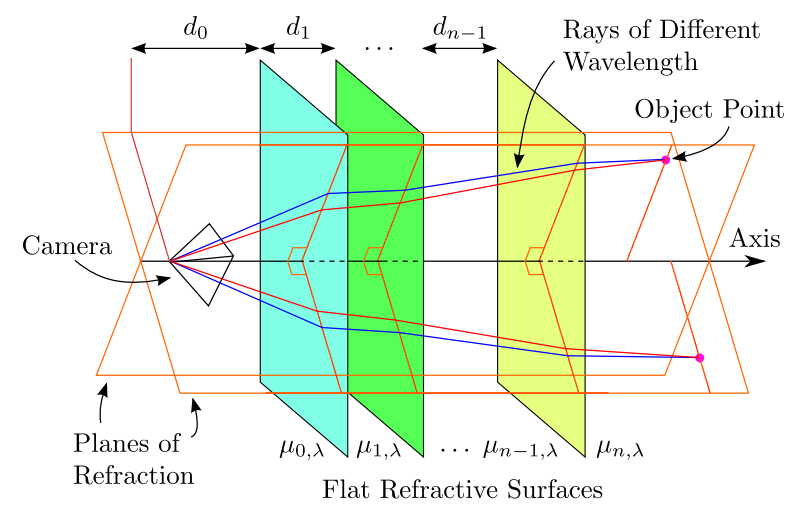

Figure 1. Flat refraction camera model with $n$ layers. The refractive index of each layer varies with the wavelength of light, resulting in a different path for each wavelength. All paths from a single object point lie on a common plane containing the refraction axis.

demonstrate how to perform the calibration in practice, including the construction of a calibration device using inexpensive parts. Lastly, we provide an experimental evaluation of our method using both simulated and real data. We develop an original procedure to obtain ground truth values for real data, which is missing in previous works on underwater camera calibration.

\section{Related work}

Treibitz et al. analyzed the case of a single refraction at a flat air-water interface, and showed that such a camera system does not have a single viewpoint. They developed a calibration procedure to find the unknown distance between the camera center and the interface, assuming that the interface is parallel to both the image plane and the checkerboard calibration pattern. The distance to the calibration pattern must also be physically measured [11].

Sedlazeck and Koch developed a more flexible calibration method that does not require a calibration object, and also accounts for two refractions when the port of the camera housing is thick. However, their method is timeconsuming because it relies heavily on nonlinear optimiza- 
tions, and the accuracy on real data is not analyzed [8].

There have been several different approaches to 3D reconstruction with explicit consideration of refraction. For example, Gedge et al. augmented the standard epipolar geometry with a refractive ray-tracing component [5]. Chang and Chen derived closed-form solutions for structure-frommotion assuming known vertical direction. Chari and Sturm showed the existence of a fundamental matrix for two cameras sharing a common refractive interface, but their analysis is theoretical and has not been developed into an algorithm [3].

A recent paper by Agrawal et al. showed that the flat refraction camera model corresponds to an axial camera. With this realization, they formulated a calibration framework that can handle multi-layer refraction models uniformly through a set of linear constraints on the model parameters. The resulting method still uses nonlinear optimization, but produces initial estimates efficiently by solving a set of linear systems while only assuming that the calibration object geometry is known [1]. Subsequently, Sedlazeck and Koch applied these results in a new analysis-bysynthesis approach coupled with an evolution algorithm for optimization [6].

Our work also builds on the framework by Agrawal et al., but unlike Sedlazeck and Koch, we do not focus on the optimization method. Instead, we examine the properties of the refraction camera model with respect to the dispersion of light, and find that by taking these properties into account, we can achieve greater calibration accuracy than if they were ignored.

\section{Flat refraction model}

We first describe the flat refraction camera model and its parameters. An example application which fits this model is a perspective camera placed in a watertight housing with a flat glass port.

Consider figure 1, which illustrates a pinhole perspective camera observing a scene through $n \geq 1$ parallel refraction layers. Each refraction layer $i=1, \ldots, n$ is defined by a thickness $d_{i}$ and a refractive index $\mu_{i, \lambda}$, which may depend on the wavelength $\lambda$ of light. The distance between the camera and the first layer is given by $d_{0}$, and the refraction axis $\mathbf{A}$ is the vector from the camera center that is perpendicular to all of the refraction layers.

The goal of calibration is to estimate some or all of these parameters. In this paper we assume that $n$ and $\mu_{i, \lambda}$ are known from the construction of the camera system and its operating environment (Agrawal et al. show how refractive indices can also be estimated [1]). Moreover, if the camera is placed in an underwater housing, the thickness of the port is often known. We therefore focus on estimating $\mathbf{A}$ and one or more layer thicknesses $d_{i}$.

Refraction at the interface between adjacent layers of dissimilar materials takes place according to Snell's law. The incident and refracted rays as well as the surface normal lie in a common plane, which means that the entire light path lies on a single "plane of refraction" [1].

\subsection{Dispersion of light}

In most common substances, the index of refraction varies with the wavelength of the incident light. (By wavelength, we mean the wavelength in a vacuum.) A single ray of polychromatic light will be refracted into multiple rays depending on the wavelength components. In the context of the flat refraction camera model, this implies that a single physical point will be imaged at multiple image points. Consequently, the location of the physical point can be triangulated given that the model parameters are known, or a constraint on the parameters can be obtained given that the point location is known.

For underwater imaging, the refracting medium is water. According to Daimon et al., distilled water at $19^{\circ} \mathrm{C}$ has a refractive index that varies from 1.332 for $656 \mathrm{~nm}$ light, to 1.343 for $404 \mathrm{~nm}$ light [4]. While the variation appears small, the resulting angular difference is sufficient to be measured by a typical consumer camera.

In the flat refraction model discussed above, for one refraction $(n=1)$ the amount of dispersion can be characterized as the solution of a quartic equation. Figure 2 (left) shows the plane of refraction for a ray passing through the refraction interface at $(0, q)$ and reaching an object point $\left(d_{1}, p+q\right)$; without loss of generality we assume $d_{0}=1$. The refractive indices for this ray are $\mu_{0, a}$ and $\mu_{1, a}$ on the left and right sides of the interface respectively. Consider another ray with different refractive indices $\mu_{0, b}, \mu_{1, b}$ passing through the same object point but refracting at a different location $(0, q+\delta)$. Snell's law gives:

$$
\begin{gathered}
\mu_{0, a} \frac{q}{\sqrt{q^{2}+1}}=\mu_{1, a} \frac{p}{\sqrt{p^{2}+d_{1}^{2}}}, \\
\mu_{0, b} \frac{q+\delta}{\sqrt{(q+\delta)^{2}+1}}=\mu_{1, b} \frac{p-\delta}{\sqrt{(p-\delta)^{2}+d_{1}^{2}}} .
\end{gathered}
$$

Squaring and rearranging (2) gives

$$
\left(\mu_{0, b}^{2}-\mu_{1, b}^{2}\right) q_{\delta}^{2} p_{\delta}^{2}+\mu_{0, b}^{2} q_{\delta}^{2} d_{1}^{2}-\mu_{1, b}^{2} p_{\delta}^{2}=0
$$

where $q_{\delta}=q+\delta$ and $p_{\delta}=p-\delta$. Solving (1) for $p$ and substituting into (3) gives a quartic equation in $\delta$ in terms of $q, d_{1}$, and the refractive indices. On the other hand, if $\delta$ and $q$ are known, then one can view this as a quadratic equation in $d_{1}$ for triangulating the object point. Figure 2 (right) shows how $\delta$ varies with $q$ and $d_{1}$ for the underwater calibration case.

For two or more refractions, analytic equations become more difficult to derive as the polynomial degree increases [1]. It is noteworthy that most transparent materials exhibit 

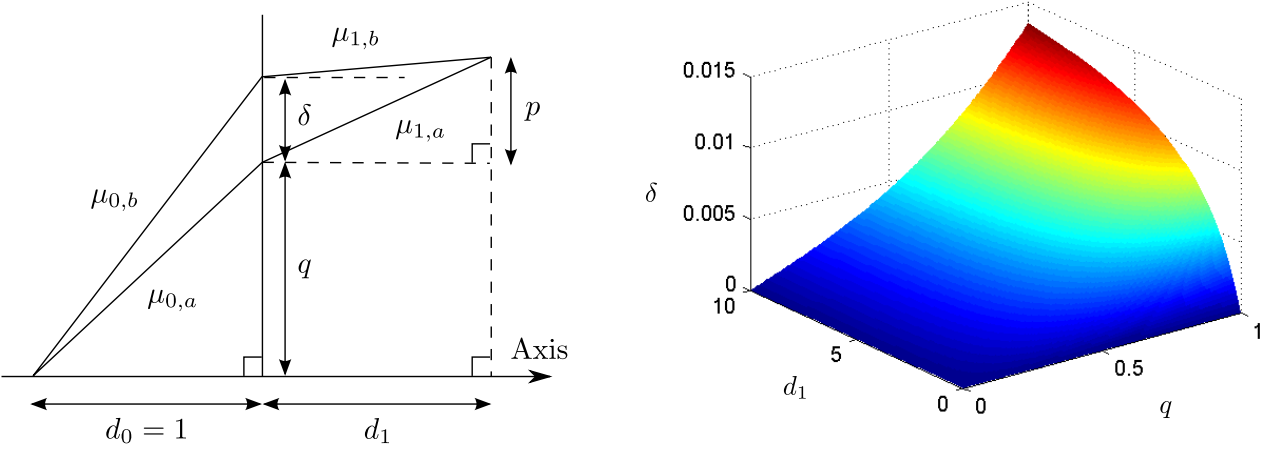

Figure 2. (Left) Geometric analysis of the dispersion, $\mu_{1, b}>\mu_{0, b}, \mu_{1, a}>\mu_{0, a}$, and $\mu_{0, b} / \mu_{1, b}<\mu_{0, a} / \mu_{1, a}$. (Right) Amount of dispersion for various settings of $q$ and $d_{1}$, for $\mu_{0, a}=\mu_{\phi b}=1, \mu_{1, a}=1332, \mu_{, b}=1343$.

"normal dispersion" where the refractive index increases as the light wavelength decreases. This implies that there will be nonzero dispersion for all rays not perpendicular to the refraction layers.

\section{Calibration}

In this section we develop constraint equations using dispersion for calibration. A key idea from Agrawal et al. is that the refraction axis $\mathbf{A}$ can be estimated first, which then allows the layer thicknesses to be found by solving a linear system of equations [1].

By considering the dispersion of light, we obtain a new and effective constraint on $\mathbf{A}$. We show how to incorporate dispersion as a form of triangulation in estimating the layer thicknesses, and also in a final nonlinear optimization step to refine the estimated parameters.

\subsection{Axis estimation}

Consider a coordinate system with the origin at the camera center, where we describe image points by ray directions from the origin. Let $\mathbf{v}_{a}, \mathbf{v}_{b}$ be unit vectors for the directions of two rays of different wavelengths $a, b$, and suppose they correspond to a single point in the scene. Both rays must lie on the same plane of refraction containing the refraction axis $\mathbf{A}$, which passes through the camera center. Therefore, if the refractive indices differ for the two wavelengths such that $\mathbf{v}_{a} \neq \mathbf{v}_{b}$, we have the following dispersion constraint:

$$
\text { Dispersion : }\left(\mathbf{v}_{a} \times \mathbf{v}_{b}\right)^{\top} \mathbf{A}=0 \text {. }
$$

Stacking two or more equations from distinct points yields a linear system, which we solve to find $\mathbf{A}$ by the method of least squares. Unlike Agrawal et al.'s work, this constraint does not involve the coordinates of scene points at all, and the residual being minimized is the sine of the angle between the axis and the plane of refraction. In contrast, the 8-point algorithm proposed by Agrawal et al. uses a single linear system to solve for both the refraction axis and the calibration object pose [1], even though the two quantities are not inherently related.

\section{Image and measurement noise}

Since the effect of dispersion can be quite small, we added a preprocessing step in our implementation to reduce the impact of random noise. Denote the image point corresponding to $\mathbf{v}_{a}$ by $\mathbf{x}$ and the point corresponding to $\mathbf{v}_{b}$ by $(\mathbf{x}+\mathbf{w})$. For convenience of notation, let image points be in $\mathbb{R}^{3}$ with zero third coordinate.

Before applying (4), we compute a new point pair $\{\mathbf{y},(\mathbf{y}+\overline{\mathbf{w}})\}$ as an "average" within a local neighborhood. Suppose we have $k$ pairs of points $\left\{\mathbf{x}_{i},\left(\mathbf{x}_{i}+\mathbf{w}_{i}\right)\right\}_{i=1: k}$ in the neighborhood, with each pair defining a line that is the projection of the corresponding plane of refraction. In the absence of noise, these lines must all pass through a point $\mathbf{u}$ where the image plane and the refraction axis intersect. Let $\overline{\mathbf{w}}=\frac{1}{k} \sum_{i=1}^{k} \mathbf{w}_{i}$ be the average line direction, then we have that

$$
\left(\mathbf{u}-\mathbf{x}_{i}\right) \times \mathbf{w}_{i}=0 \forall i \Rightarrow \mathbf{u} \times \overline{\mathbf{w}}=\frac{1}{k} \sum_{i=1}^{k} \mathbf{x}_{i} \times \mathbf{w}_{i}
$$

where the implication follows by summing over $i$. Now we constrain the line $\{\mathbf{y},(\mathbf{y}+\overline{\mathbf{w}})\}$ to also pass through $\mathbf{u}$ :

$$
(\mathbf{u}-\mathbf{y}) \times \overline{\mathbf{w}}=0 \Rightarrow \mathbf{u} \times \overline{\mathbf{w}}=\mathbf{y} \times \overline{\mathbf{w}} .
$$

Substituting (6) into (5) eliminates $\mathbf{u}$ and gives a linear equation for $\mathbf{y}$. There are infinitely many solutions, so we find one that is "close" to the original point pair by choosing $\mathbf{y}$ on the line perpendicular to $\overline{\mathbf{w}}$ and passing through the centroid of the point neighborhood. The image points $\{\mathbf{y},(\mathbf{y}+\overline{\mathbf{w}})\}$ are then back-projected into rays and used in the dispersion constraint.

In both our simulated and real data experiments we found that that this averaging procedure works very well. The neighborhood radius was set manually to $6 \%$ of the image width, typically encompassing around 20 point pairs. 


\subsection{Layer thicknesses}

Suppose that the geometry of the points $\mathbf{P}$ on the calibration object are known. With appropriate estimates of the refraction axis $\mathbf{A}$, object rotation $R$, and object translation $\mathbf{t}_{\perp}$ in the plane perpendicular to the axis, Agrawal et al. showed that the thicknesses of the refraction layers can be formulated as a linear system (Eqn. (9) in [1]).

We follow their method, but incorporate a form of triangulation through the use of multiple wavelengths of light. As discussed in Section 4.1, a single object point projects to multiple image points when dispersion is present. More formally, if $F$ is a function that projects a $3 \mathrm{D}$ point $\mathbf{X}$ to a point $\mathbf{q}$ on the refractive surface closest to the camera, then for two different wavelengths $a$ and $b$ we define the wavelength triangulation constraint:

$$
\text { Triangulation: } \quad\left\{\begin{array}{l}
\mathbf{q}_{a}=F\left(\mathbf{X}, \mu_{0, a}, \ldots, \mu_{n, a}\right) \\
\mathbf{q}_{b}=F\left(\mathbf{X}, \mu_{0, b}, \ldots, \mu_{n, b}\right)
\end{array}\right.
$$

where the $d_{i}$ and $\mathbf{A}$ parameters are the same in both cases and are omitted for clarity. This constraint is imposed simply by including all rays for each object point in the linear system for layer thicknesses (as well as in the linear system for recovering object pose, described below). The geometry is depicted in Figure 1, and Figure 3 (bottom left) displays an actual photograph of object points under the influence of dispersion.

\section{Recovering object pose}

In Agrawal et al.'s solution for finding the refraction layer thicknesses, the required object pose parameters $R$ and $\mathbf{t}_{\perp}$ are computed together with $\mathbf{A}$ through the "coplanarity constraint" (Eqn. (5) in [1]). We show that the constraint equations can be simplified significantly to obtain these parameters by assuming that $\mathbf{A}$ is known (see Section 4.1). The coplanarity constraint states that each camera ray $\mathbf{v}$ and its corresponding object point $\mathbf{P}$, after being transformed into camera coordinates, must lie on a plane of refraction containing the refraction axis:

$$
\begin{array}{r}
(R \mathbf{P}+\mathbf{t})^{\top}(\mathbf{A} \times \mathbf{v})=0 \\
\Leftrightarrow \underbrace{\left[\begin{array}{ll}
\mathbf{P}^{\top} \otimes \mathbf{v}^{\top} & \mathbf{v}^{\top}
\end{array}\right]}_{B}\left[\begin{array}{c}
E_{(1: 9)} \\
\mathbf{s}
\end{array}\right]=0
\end{array}
$$

where $\otimes$ denotes the Kronecker product, $E=[\mathbf{A}]_{\times} R$ and $\mathbf{s}=\mathbf{A} \times \mathbf{t}$, and subscripts in parentheses denote matrix elements taken column-wise. Since the refraction axis is known, we can rotate the coordinate system such that $\mathbf{A}$ is aligned with the positive z-axis. Let $r_{1}, \ldots r_{9}$ be the entries of $R$, then with such a transformation we have

$$
E=\left[\begin{array}{ccc}
-r_{2} & -r_{5} & -r_{8} \\
r_{1} & r_{4} & r_{7} \\
0 & 0 & 0
\end{array}\right], \mathbf{s}=\left[\begin{array}{lll}
-t_{2} & t_{1} & 0
\end{array}\right]^{\top} .
$$

Furthermore, for a planar calibration object such as a checkerboard where $\mathbf{P}_{(3)}=0$, columns 7-9 of $B$ are zero (Eqn. (9)). Therefore we can drop the last row and the last column of $E$ to obtain the simplified constraint:

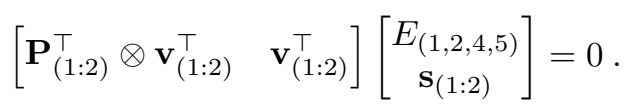

Stacking five or more equations and solving the resulting linear system yields four entries of $R$ and the translation perpendicular to the axis, up to an unknown scale factor. We recover the scale factor and the remaining entries of $R$ by solving the quadratic constraint that this is an orthogonal matrix.

In general there are four possible solutions. The sign of the scale factor corresponds to one solution in front of the camera and one behind. Two further solutions are obtained by negating the signs of $r_{8}$ and $r_{7}$, corresponding to a reflection across the plane parallel to the refraction layers and passing through the object origin. The correct solution is found after estimating the refraction layer thicknesses by choosing the one with the minimum reprojection error.

\subsection{Nonlinear refinement}

The estimated refraction axis $\mathbf{A}$, layer thicknesses $d_{i}$, as well as object pose parameters $R$ and $\mathbf{t}$ are refined by a nonlinear optimization. The objective function to minimize is the root-mean-squared Euclidean distance reprojection error, where each object point is reprojected using all of the observed wavelengths. Therefore, the optimization implicitly tries to satisfy wavelength triangulation constraint as defined in Section 4.2. We use the MATLAB function lsqnonlin to perform the optimization.

A noteworthy difference between our procedure and that of Agrawal et al. is that we do not solve the analytical forward projection equations, which for two refractions involve $12^{\text {th }}$ degree polynomials [1]. Instead, for a given object point, we first compute the plane of refraction. Then we perform a 1D bisection search for the angle of a camera ray on that plane such that the back-projected ray intersects the given point. This procedure is accurate and adaptable to an arbitrary number of refractions. Moreover, it is reasonably fast and can be easily parallelized to handle many points at once.

\section{Implementation}

\subsection{Calibration object}

A standard checkerboard pattern does not suffice for our method because the dispersion effect is not readily apparent under normal lighting conditions. We designed and built a new device that illuminates a perforated grid with two distinct wavelengths of light, forming a precisely known pat- 

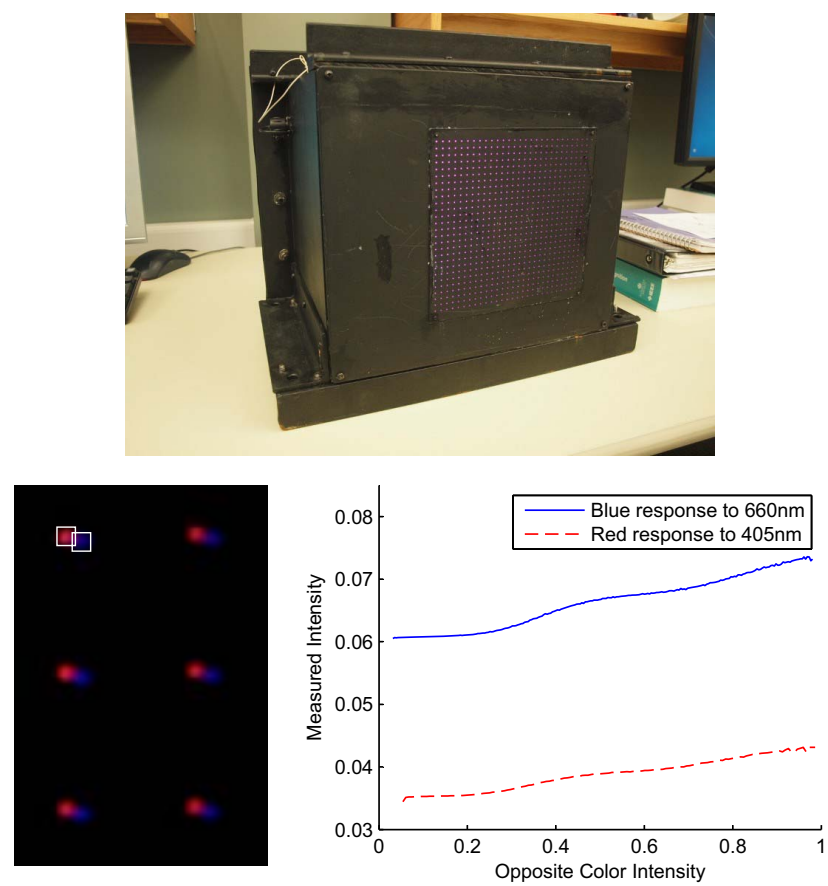

Figure 3. (Top) Calibration device. (Bottom Left) Close-up of the illuminated points viewed through an air-water interface (best viewed in color; one pair of points marked for visibility). The dispersion seen here is typical at around 6-7 pixels. (Bottom Right) Influence of red light on blue pixels and vice versa. The top curve shows the average intensity of a blue pixel as a function of the intensity of the neighboring red pixels when the camera observes a $660 \mathrm{~nm}$ light source; similarly for the bottom curve for red pixels.

tern of point light sources, each of which produces a measurable amount of dispersion.

As shown in Figure 3, this device consists of a watertight acrylic enclosure containing 122 LEDs arranged in a grid pattern. Half of the LEDs emit 660nm light ${ }^{1}$ and the other half $405 \mathrm{~nm}$ light $^{2}$, with a series of diffuser films blending them together. The dominant wavelengths of these LEDs were confirmed by a spectrometer. The light travels through one transparent side of the enclosure, in front of which we mounted a precision CNC-drilled plate with a $27 \times 29$ grid of $0.65 \mathrm{~mm}$ diameter holes spaced $6 \mathrm{~mm}$ apart. The device is powered by a lithium polymer battery installed inside the enclosure.

We chose the two light wavelengths to be as far apart as possible to maximize the amount of dispersion, while remaining visible to typical cameras equipped with CCD or CMOS sensors and a Bayer-pattern color filter array (CFA). Longer wavelengths in the infrared range suffer from high attenuation in water, while shorter wavelengths are not readily available for LEDs and may pose a hazard to the user.

\footnotetext{
${ }^{1}$ Lumex SSL-LX5093SRC/E

${ }^{2}$ Bivar UV5TZ-405-30
}

\subsection{Point localization}

Our calibration object provides point light sources emitting two wavelengths of light simultaneously. Using color cameras with standard Bayer-pattern CFAs, we can isolate the two wavelengths by considering the blue and the red pixels separately, before demosaicing has been applied. Our tests indicate that the red pixel sensitivity to $405 \mathrm{~nm}$ light is less than $1 \%$, and the blue pixel sensitivity to $660 \mathrm{~nm}$ light is less than $1.5 \%$ (see Figure 3). Thus we consider the correlation between the two color channels to be negligible.

The centers of the image points are estimated under the assumption that the point spread function is a bivariate Gaussian. For each point we compute the Gaussian parameters from the covariance matrix of pixel intensities, and refine the estimate using a nonlinear optimization. The details are omitted due to space constraints.

\subsection{Lens chromatic aberrations}

An important consideration with refraction-based lenses is that dispersion also occurs within the lens elements, the effects of which are known as chromatic aberrations (CA). It is necessary to correct for $\mathrm{CA}$ in order to isolate the dispersive effect of the refraction planes in front of the camera.

For this purpose, we use the image distortion model introduced by Brown [2]. We first capture images of our calibration object in air, and obtain two sets of camera intrinsic parameters using the red and the blue channels separately. Then we apply a nonlinear optimization to adjust the image distortion parameters of the blue channel so that the blue feature points are aligned with the red feature points. The choice of which color channel to warp is arbitrary as long as we do so consistently with the underwater images.

\section{Results}

\subsection{Synthetic data}

For our synthetic data experiments we simulated a camera with a resolution of $4368 \times 2912$ pixels and a focal length of 4633 pixels, which is based on the intrinsic parameters of the camera used in our real experiments. We performed experiments for the refraction model configurations listed in Table 1. All refractive indices are assumed known and are listed in table 2.

\begin{tabular}{|c|c|c|c|c|c|}
\hline & Refractions & $d_{0}$ & Est.? & $d_{1}$ & Est.? \\
\hline \hline a) & Air $\rightarrow$ Water & 60 & Yes & - & - \\
b) & Air $\rightarrow$ Acrylic $\rightarrow$ Water & 60 & Yes & 5.6 & No \\
c) & Air $\rightarrow$ Acrylic $\rightarrow$ Water & 60 & Yes & 30 & Yes \\
\hline
\end{tabular}

Table 1. Configurations for synthetic data experiments, showing the values for parameters $d_{0}$ and $d_{1}$ and whether they were estimated during calibration or assumed known. The angle between the refraction axis and the camera's optical axis was set to 4.47 degrees for all configurations. 

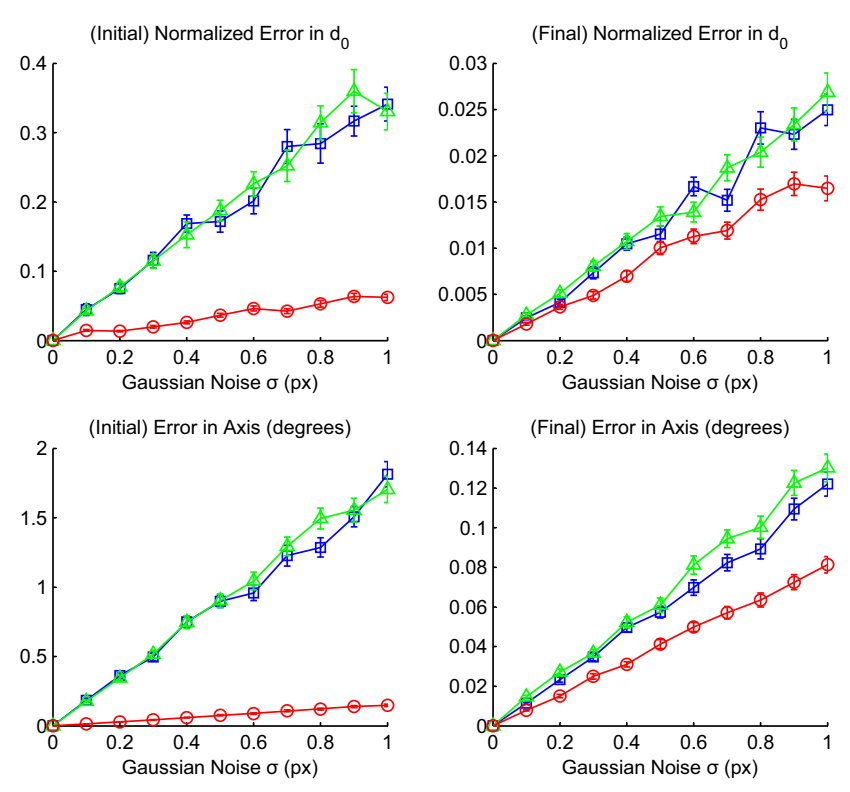

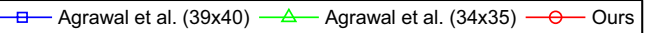

Figure 4. Synthetic data results for configuration a). Error bars represent standard error. Results for configuration b) are very similar and are omitted. (Left column) Before nonlinear refinement. (Right column) After nonlinear refinement.

\begin{tabular}{|c|c|c|}
\hline Wavelength & Water & Acrylic \\
\hline \hline $660 \mathrm{~nm}$ & 1.33151 & 1.488 \\
\hline $405 \mathrm{~nm}$ & 1.34318 & 1.516 \\
\hline Unspecified/589nm & 1.33344 & 1.491 \\
\hline
\end{tabular}

Table 2. Refractive indices used in all experiments. Sources are [4] for water and [7] for acrylic.

The calibration pattern was a $27 \times 29$ planar grid of points emitting both $405 \mathrm{~nm}$ and $660 \mathrm{~nm}$ light. Since the use of two wavelengths in our method could be construed as doubling the number of points, to provide a fair comparison we used a $39 \times 40$ grid with Agrawal et al.'s method; moreover, all the points used $405 \mathrm{~nm}$ light to remove any advantage due to stronger refractions. For a more direct comparison with our real data results, we also included a dataset for a $34 \times 35$ grid to show the impact of using a smaller number of points.

All feature points were perturbed by random Gaussian noise with standard deviation ranging from 0 to 1 pixel. For each noise level we generated 100 trials with the calibration pattern placed 440 units in front of the camera and rotated randomly by up to 20 degrees.

Figures 4 and 5 summarize the results. We include results before the nonlinear refinement step to show the effectiveness of the dispersion and wavelength triangulation constraints. For Agrawal et al.'s method the corresponding estimates are computed by the 8-point algorithm and the linear system for recovering layer thicknesses.
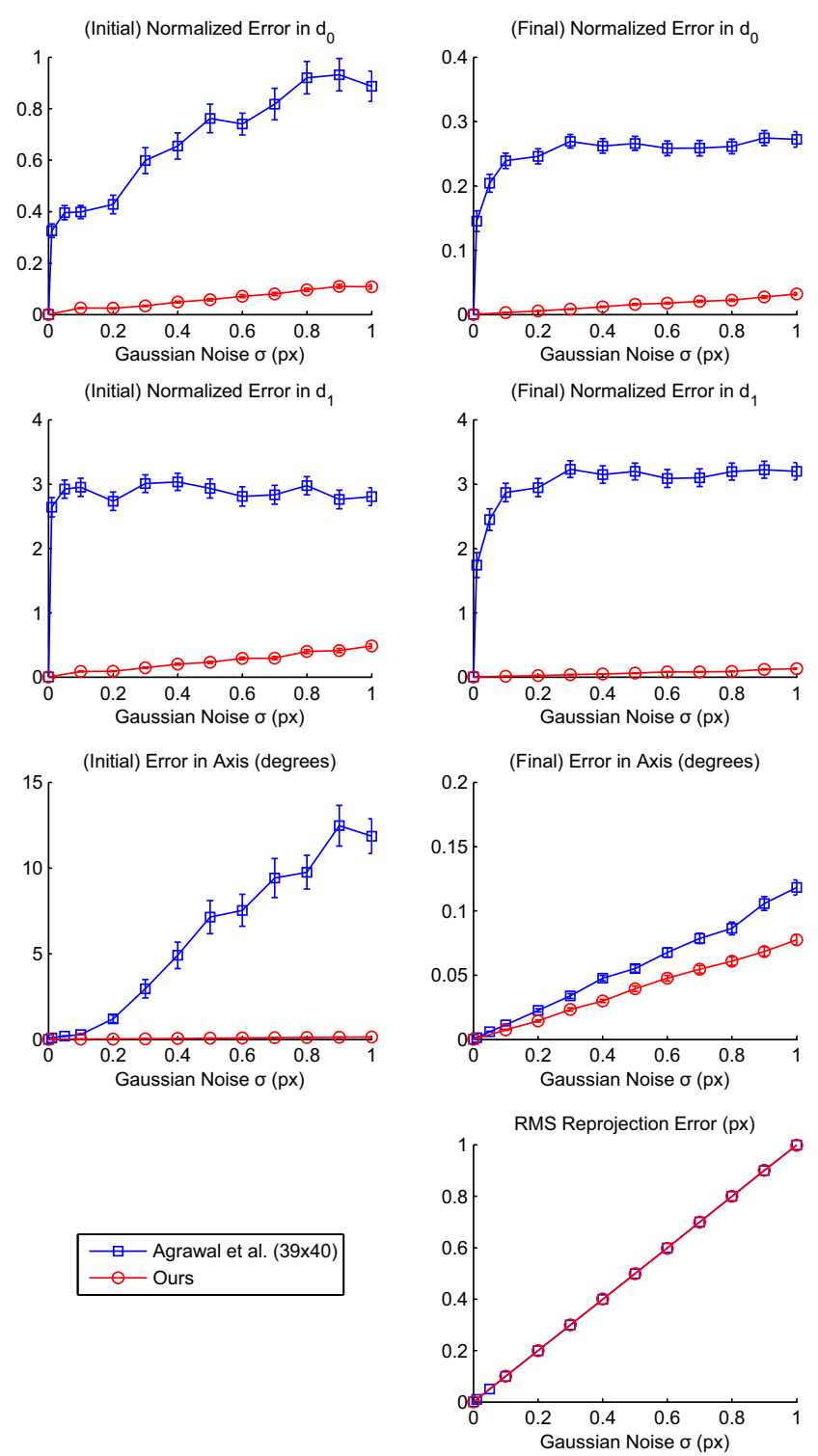

Figure 5. Synthetic data results for configuration c). Error bars represent standard error. Plots for Agrawal et al.'s method contain two additional data points at $\sigma=0.01,0.05$. (Left column) Initial estimates before nonlinear refinement. (Right column) After nonlinear refinement.

While our method gives more accurate results overall, the difference is particularly striking for configuration c). The layer thickness error plots for Agrawal et al.'s method rise sharply as the noise increases and the final estimates are far from the ground truth. We believe that the refraction model is correct because the error goes to zero in the absence of noise, the refraction axis estimates appear reasonable, and the reprojection error is being minimized properly. Unfortunately, Agrawal et al.'s work [1] does not give layer thickness estimation results for this configuration for us to corroborate our findings. 


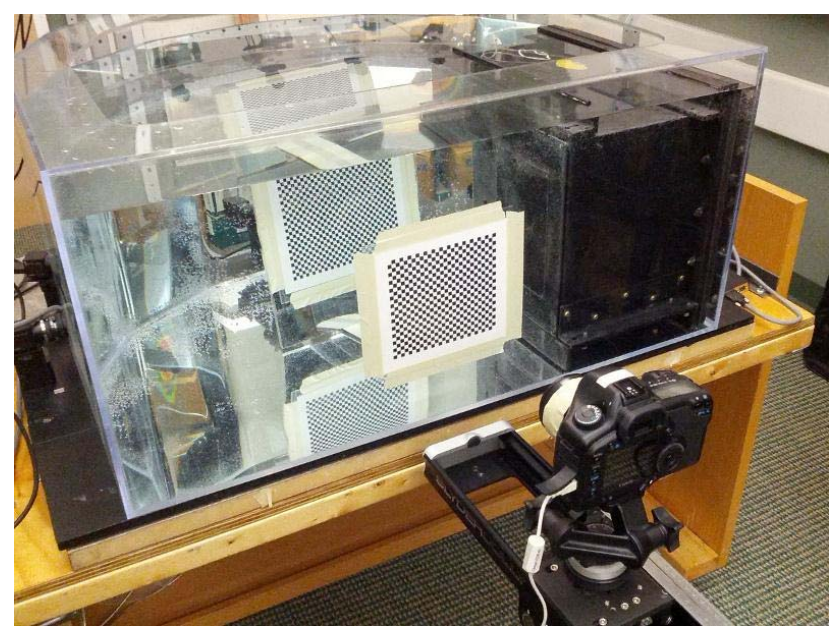

Figure 6. Experimental setup, showing the calibration device and a checkerboard inside the tank, a checkerboard affixed to the tank surface, and the camera mounted on a SlyderDolly translation rail.

In light of our results, we believe that the additional constraints provided by dispersion and wavelength triangulation have a significant impact on calibration accuracy beyond simply doubling the number of feature points.

\subsection{Real data}

For our real experiments we used a Canon 5D camera with a resolution of $4368 \times 2912$ pixels and a $30 \mathrm{~mm}$ fixed focal length lens. The camera was calibrated offline for both the intrinsic parameters and chromatic aberration correction (see 5.3).

We collected two sets of data, one using our novel calibration device with a $27 \times 29$ grid of points, and a second using a $34 \times 35$ checkerboard pattern for comparison with Agrawal et al.'s method. (Because the squares cannot be too small, this checkerboard has somewhat fewer points than in our synthetic data experiments. The results in Fig. 4 show that the impact of using fewer points with Agrawal et al.'s method is minimal.) The calibration objects were placed inside an acrylic water tank approximately $45 \mathrm{~cm}$ behind the front surface and moved around slightly within the camera's field of view. The camera was located within $10 \mathrm{~cm}$ of the tank surface. We used an air $\rightarrow$ acrylic $\rightarrow$ water refraction model in all calibrations, with the tank wall thickness assumed known at a measured value of $5.6 \mathrm{~mm}$. Figure 6 shows a photo of the experimental setup, and the calibration results are shown in Figure 7 and Table 3.

Although our method gives accurate results, we noticed that the nonlinear refinement step did not improve the estimate of the refraction axis, which was very close to the ground truth to begin with (see Figure 7). Therefore we tried a "fixed axis" variation of our method in which the estimated axis is fixed during the nonlinear optimization step. Unfortunately this caused the error in $d_{0}$ to increase. Since these observations disagree with our results using synthetic data, we attribute the source of error to measurement noise, lens distortions, and/or the pinhole camera approximation. We plan to investigate this issue further in our future work.

Interestingly, we found that the estimated $d_{0}$ was particularly sensitive to variations in the refractive index difference $\lambda_{2,405 \mathrm{~nm}}-\lambda_{2,660 \mathrm{~nm}}$ for water, but much less sensitive to variations in both index values that do not change this difference. This is in line with the theory since the triangulation constraint is based on the difference in refraction angle. In our experiments, we found that varying only one index value by 0.0005 affected the estimated $d_{0}$ by about $4 \mathrm{~mm}$, whereas varying both values correspondingly by this amount had an effect of only $0.3 \mathrm{~mm}$.

\subsubsection{Obtaining ground truth}

Because the camera is focused on objects within the tank, it is not possible to obtain a direct measurement of the front surface of the tank. Instead, we mounted our camera on a SlyderDolly translation rail, which allowed us to move the camera precisely in a straight line. We also attached a ruler with a needle to accurately measure the camera's motion along the rail.

The ground truth values for the calibration parameters were determined after the datasets were captured. Our procedure was as follows:

1. Measure the initial position of the camera as used for dataset capture.

2. Translate the camera backwards until the tank surface is just in focus.

3. Affix a checkerboard pattern on the tank surface, in the center of the camera's field of view. (The thickness of the checkerboard is negligible.)

4. Capture a set of 48 images, translating the camera backwards by $2 \mathrm{~mm}$ each time.

Each of the 48 images yielded a measurement of the pose of the tank surface with respect to the camera. The refraction model parameters were then determined in two steps. Firstly, since the camera orientation is not changed by linear motion, we averaged the rotation measurements to obtain the ground truth for the refraction axis. Secondly, the initial position of the camera was extrapolated from the 48 pose measurements. We did so by fitting a line to the data points, together with a $2 \mathrm{~mm}$ scale that minimized squared distance error. The parameter $d_{0}$ was then computed as the initial camera translation in the axis direction.

\section{Conclusion and future work}

We have described a novel method for calibrating the geometry of a camera observing a scene through one or more 


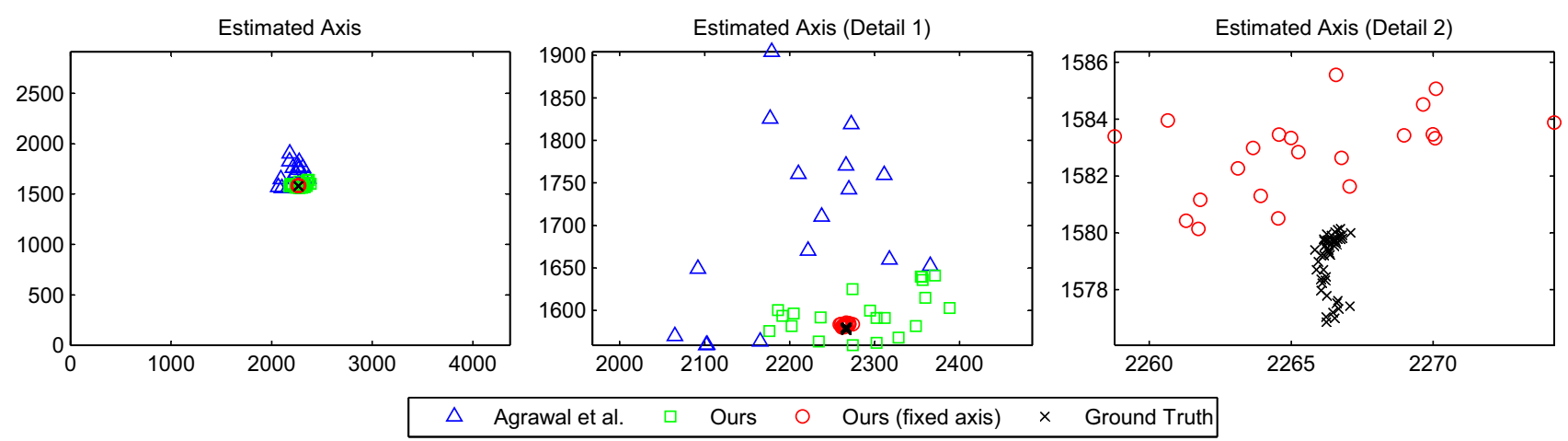

Figure 7. Refraction axis estimation results. Each marker represents the point of intersection between the axis and the image plane. The vertical and horizontal axes are in pixels and are shown at the same scale. (Left) The entire image. (Middle) Detail near the center of the image. (Right) Detail showing the distribution of ground truth estimates and the estimates from our method.

\begin{tabular}{|c|c|c|c|c|}
\hline Method & Estimated $d_{0}(\mathrm{~mm})$ & $\sigma$ & Error in Axis A (degrees) & $\sigma$ \\
\hline \hline Ground Truth & 45.91 & $0.07^{\dagger}$ & $0.011^{\ddagger}$ & 0.006 \\
\hline Agrawal et al. & 43.34 & 39.22 & 2.173 & 0.775 \\
\hline Ours & 46.09 & 11.77 & 0.866 & 0.393 \\
Ours (fixed axis) & 52.31 & 14.08 & 0.065 & 0.022 \\
\hline
\end{tabular}

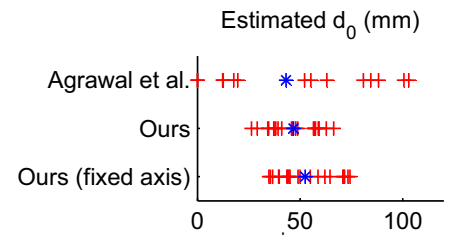

Table 3. Estimated calibration parameters using real data. ${ }^{\dagger}$ Standard deviation of linear scale fitting error (Sec. 6.2 .1 ). ${ }^{\ddagger}$ Error of each measurement point with respect to the average. (Inset) Scatterplot of the estimated $d_{0}$ values and mean values.

flat refraction layers. Our method exploits the effect of dispersion, which has previously been ignored, to develop constraints on the calibration parameters by using multiple wavelengths of light. We have shown that these constraints are analogous to triangulation, and have demonstrated using synthetic and real experiments that they can improve the accuracy of the calibration.

In the future we plan to extend our method to calibrate multiple cameras simultaneously, and apply the results to $3 \mathrm{D}$ reconstruction with explicit modeling of refraction. It will be interesting to compare the real-world accuracy of such a method with standard methods that do not model refraction. Additionally, with appropriate scene illumination techniques, the wavelength triangulation constraint may be directly applicable in single-view $3 \mathrm{D}$ reconstruction or as a means to improve multi-view reconstruction quality.

Another direction to investigate is calibration with unknown refractive indices, where the dispersion effect may aid in recovering these unknowns.

\section{Acknowledgements}

We thank the reviewers for their constructive comments, NSERC for the financial support, and Steve Sutphen for building the calibration device. We also thank the Computer Graphics Group members for their comments.

\section{References}

[1] A. Agrawal, S. Ramalingam, Y. Taguchi, and V. Chari. A theory of multi-layer flat refractive geometry. In CVPR, 2012.
$1,2,3,4,6$

[2] D. C. Brown. Close-range camera calibration. Photogrammetric Engineering, 37:855-866, 1971. 5

[3] V. Chari and P. Sturm. Multi-view geometry of the refractive plane. In $B M V C, 2009.2$

[4] M. Daimon and A. Masumura. Measurement of the refractive index of distilled water from the near-infrared region to the ultraviolet region. Appl. Opt., 46(18):3811-3820, 2007. 2,6

[5] J. Gedge, M. Gong, and Y.-H. Yang. Refractive epipolar geometry for underwater stereo matching. In $C R V$, pages 146-152, 2011. 1, 2

[6] A. Jordt-Sedlazeck and R. Koch. Refractive calibration of underwater cameras. In ECCV (5), volume 7576 of Lecture Notes in Computer Science, pages 846-859. Springer, 2012. 1,2

[7] S. N. Kasarova, N. G. Sultanova, C. D. Ivanov, and I. D. Nikolov. Analysis of the dispersion of optical plastic materials. Optical Materials, 29(11):1481 - 1490, 2007. 6

[8] A. Sedlazeck and R. Koch. Calibration of housing parameters for underwater stereo-camera rigs. In BMVC, 2011. 2

[9] A. Sedlazeck and R. Koch. Perspective and non-perspective camera models in underwater imaging - overview and error analysis. In Theoretical Foundations of Computer Vision, volume 7474 of Lecture Notes in Computer Science, pages 212-242. Springer, 2011. 1

[10] M. Shortis, E. Harvey, and J. Seager. A review of the status and trends in underwater videometric measurement. SPIE Conf. 6491, Videometrics IX, 2007. 1

[11] T. Treibitz, Y. Y. Schechner, and H. Singh. Flat refractive geometry. In CVPR, 2008. 1 